\title{
How Does Chronic Back Pain Influence Quality of Life in Koreans: A Cross-Sectional Study
}

\author{
Yong Soo Choi ${ }^{1}$, Dong Jun Kim ${ }^{2}$, Kyu Yeol Lee ${ }^{3}$, Ye Soo Park ${ }^{4}$, Kyu Jung Cho ${ }^{5}$, \\ Jae Hyup Lee ${ }^{6}$, Hyou Young Rhim ${ }^{7}$, Byung Joon Shin ${ }^{8}$ \\ ${ }^{1}$ Department of Orthopedic Surgery, Kwangju Christian Hospital, Gwangju, Korea \\ ${ }^{2}$ Department of Orthopedic Surgery, Ewha Womans University School of Medicine, Seoul, Korea \\ ${ }^{3}$ Department of Orthopedic Surgery, Dong-A University College of Medicine, Busan, Korea \\ ${ }^{4}$ Department of Orthopaedic Surgery, Hanyang University Guri Hospital, Hanyang University College of Medicine, Guri, Korea \\ ${ }^{5}$ Department of Orthopedic Surgery, Inha University School of Medicine, Incheon, Korea \\ ${ }^{6}$ Department of Orthopedic Surgery, Seoul National University College of Medicine, Seoul, Korea \\ ${ }^{7}$ Department of Clinical Research, Janssen Korea, Seoul, Korea \\ ${ }^{8}$ Department of Orthopaedic Surgery, Soonchunhyang University College of Medicine, Seoul, Korea
}

\section{Study Design: A cross-sectional study.}

Purpose: To explore the impact of chronic low back pain (CLBP) on individuals' quality of life; to understand current treatment practices and level of satisfaction with treatment in patients with CLBP.

Overview of Literature: Assessing subjective, patient-reported outcomes such as quality of life is essential to health care research. Methods: Influences of the CLBP were analyzed via a questionnaire, which contained the character of CLBP, effect of pain management, Korean version Oswestry Disability Index (K-ODI) and Korean version of 12-item Short Form Health Survey (SF-12v2).

Results: Of 3,121 subjects who responded, $67.3 \%$ had moderate to severe pain; $43.5 \%$ presented prolonged CLBP of more than two years; and $32.4 \%$ had suffered from sleep disturbance due to pain. $22.8 \%$ of the patients were not satisfied with current pain management. The mean K-ODI score was 37.63; and it was positively correlated with the mean pain intensity $(r=0.6, p<0.001)$. The SF-12v2 result was negatively correlated with mean pain intensity (PCS: $r=-0.5, p<0.001$; MCS: $r=-0.4, p<0.001$ ) and also negatively correlated with the K-ODI score (PCS: $r=-0.75, p<0.001$; MCS: $r=-0.5, p<0.001$ ). The conformity between patients and doctors in pain assessment was fair $(\mathrm{K}=0.2463)$.

Conclusions: CLBP negatively affects quality of life. Of total $22.8 \%$ of the patients were not satisfied with current pain management. Such needs to be taken more seriously by doctors for improvement of satisfaction and quality of life in patients with CLBP.

Keywords: Chronic low back pain; Assessment; Quality of life

\section{Introduction}

Low back pain (LBP) and associated disabilities constitute a growing public health concern, particularly in industrialized countries [1]. It is one of the three most common reasons for healthcare visits, with an episode in presumably up to $75 \%$ of the population at some point [2]. Most cases of LBP are not severe and resolves within a few days or within few weeks; but for about $6 \%$ to $10 \%$ of patients, the disease may become recurrent or chronic $[3,4]$.

Received Jan 9, 2013; Revised May 18, 2013; Accepted May 21, 2013

Corresponding author: Byung-Joon Shin

Department of Orthopedics, Soonchunhyang University Hospital, Soonchunhyang University College of Medicine,

59 Daesagwan-ro, Yongsan-gu, Seoul 140-743, Korea

Tel: +82-2-709-9051, Fax: +82-2-796-3682, E-mail: schsbj@schmc.ac.kr

*This article was presented at the 29th Spring Congress Korean Society of Spine Surgery, 2012. 
Chronic low back pain (CLBP) is an entity developing in patients with LBP which persists for more than 12 weeks, such that recovery is slow and uncertain [4]. Chronic low back pain is often poorly understood and difficult to treat and can have a significant influence on the patient's quality of life in various areas of everyday functioning. Chronic pain is a common condition, for which patients seek care from various health care providers. It causes much suffering and disability and is frequently mistreated or undertreated [5]. As the importance of pain control is emphasized, the Agency for Health Care Policy and Research, a US agency, has issued recommendations for the treatment of pain. Health care providers should view good pain control as a source of pride and a major responsibility in quality care [6].

In 2007, characteristics and management patterns of CLBP were investigated nationally by the Korean Society of Spinal Surgery. The society reported that $65 \%$ of patients were satisfied with current pain management. Unlike in the past, recent pain management has replaced nonsteroidal anti-inflammatory drugs (NSAIDs) by more powerful analgesics. With this change of treatment pattern, the purpose of this study is to evaluate the status of CLBP management by investigating the satisfaction with current pain management in patients with CLBP. Secondary purpose of this study is to document pain intensity evaluated by patients and doctors and to assess the disability and limitation of quality of life due to CLBP.

\section{Materials and Methods}

Patient recruitment and data collection were performed by 76 physicians at 62 outpatient clinical centers, of which 53 were general hospitals and 9 were specialized spine hospitals in the Republic of Korea. The study was conducted from October 2011 to January 2012; and 3,345 patients who had visited orthopedic outpatient centers for low back pain treatment were recruited. The inclusion criteria were adult male or female patients of twenty-years or older, with CLBP symptom duration of at least three months and whose pain was controlled with analgesics at the time of the survey. Here we defined chronic pain patient as: a patient whose pain has lasted for three months or longer; who experienced continuous pain within the last one week; and/or currently taking analgesics for pain treatment. Also the patients who were capable of understanding the questionnaire and who provided written informed consent were recruited. The exclusion criteria were patients who had spinal fracture within six months and spinal tumor or other malignancy, who had undergone spinal surgery within three months, who were taking medicine for psychiatric disorder and who had possibility of exaggerated complaints due to automobile or accident insurance.

After obtaining patient consent, patients responded to questionnaires on characteristics of CLBP, Korean version of Oswestry Disability Index (K-ODI) (Suppl. 1) and quality of life by Korean version of Short Form 12 Health Survey V2 (SF-12v2) (Suppl. 2).

The ODI was originally developed as a self-assessment score for low back pain patients, and is based on patient's subjective impression of his or her own state of disability. Though the questionnaire is rather dated, it has been validated, and its reliability and sensitivity to changes in functional status have been documented $[7,8]$. It is comprised of ten items, each of which is followed by six alternatives [7]. Each question is scored from 0 to 5, and the sum of the scores is then presented as percentage. Higher score of K-ODI means that the function has decreased.

The SF-12v2 is a multipurpose short-form survey with twelve questions, all selected from the SF-36 Health Survey (Ware, Kosinski, and Keller, 1996). It is consisted of eight domains, and the values in each domain contribute to physical and mental values (physical component score [PCS] and mental component score [MCS]), which provide glimpses into mental and physical functioning and overall health-related quality of life. PCS and MCS are computed using the score of twelve questions and range from 0 to 100 , where 0 indicates the lowest level of health and 100 indicates the highest level of health [9].

Mean pain intensity was estimated by numeric rating scale (NRS), categorized into four groups: 0, pain free; $1-3$, mild; $4-6$, moderate; and 7-10, severe. The patient's satisfaction with current pain management was measured using a four-point Likert scale (very satisfied, satisfied, unsatisfied, very unsatisfied), and it was categorized into two groups (satisfaction group,very satisfied and satisfied; dissatisfaction group, unsatisfied and very unsatisfied).

Also main causative diagnosis of CLBP and current pain management, the degree of pain improvement, pain intensity, nature of pain and next treatment management plan were taken from doctors who managed patients with CLBP.

Demographic data is described with means \pm standard deviations, median, minimum, maximum and ranges by descriptive statistics. The relationship between mean pain 
intensity and satisfaction with current pain management was evaluated by chi-square test. The relationship between mean pain intensity and K-ODI score was evaluated by analysis of variance (ANOVA) test. Pearson correlation coefficients were used for correlation analysis between mean pain intensity and other variables (SF-12v2, K-ODI). Kappa statistics were used to analyze conformity of mean pain intensity evaluation between patient and doctor. Wilcoxon sum test was used for analysis of difference between pain improvement evaluation between doctors and patients.

\section{Results}

\section{Demographics}

Two hundred and twenty-four patients were excluded (62 patients were under 19 years; 38 patients had LBP less than three months; and 169 patients did not complete the questionnaire). Overall data of 3,121 patients could be evaluated; and 1,997 (64.0\%) were female. Mean age was $60.7 \pm 14.0$. The vast majority was between 50 years and 70 years, over 50 years $(2,492 / 3,121,79.8 \%)$, and 60 s $(867,27.8 \%)$ (Table 1). The main diagnoses of patients with CLBP were spinal stenosis (47.31\%) and herniated nucleus pulposus (23.52\%) (Fig. 1).

\section{Patient assessment}

Nearly half of the patients $(1,359,43.5 \%)$ had prolonged LBP for at least two years (Fig. 2). The time during the day that the patients most frequently suffered LBP was from 7 PM to midnight (1,027, 35.1\%) (Fig. 3). 32.4\%
Table 1. Demographics of patients

\begin{tabular}{lcc} 
Value & Number & Ratio \\
Sex & & \\
Male & 1,124 & 36 \\
\hline Female & 1,997 & 64 \\
\hline Age & & \\
\hline Mean \pm standard deviation (yr) & $60.7 \pm 14.0$ & - \\
\hline Median & 63 & - \\
\hline Minimun-maximum & $20-96$ & - \\
\hline $20-30$ yr & 107 & 3.4 \\
\hline $31-40$ yr & 165 & 5.3 \\
\hline $41-50$ yr & 357 & 11.4 \\
\hline $51-60$ yr & 693 & 22.2 \\
\hline $61-70$ yr & 867 & 27.8 \\
\hline $71-80$ yr & 767 & 24.6 \\
\hline$>81$ yr & 165 & 5.3 \\
\hline Total & 3,121 & 100 \\
\hline
\end{tabular}

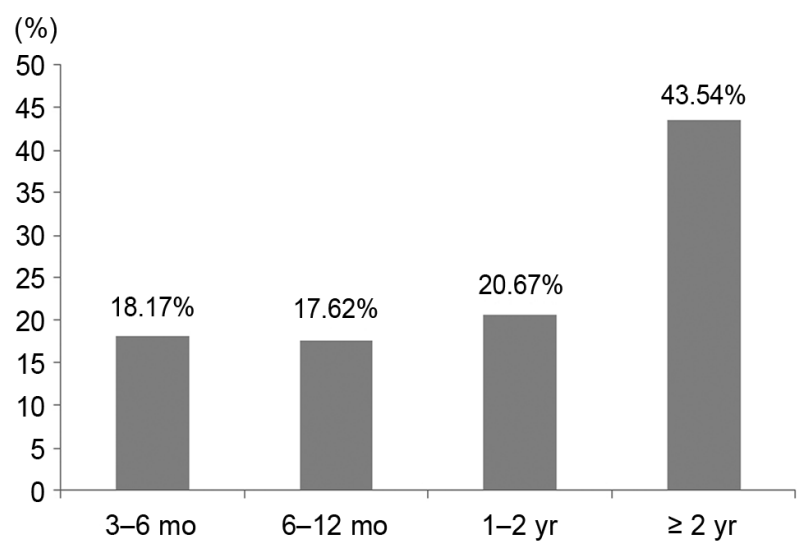

Fig. 2. Duration of chronic low back pain.

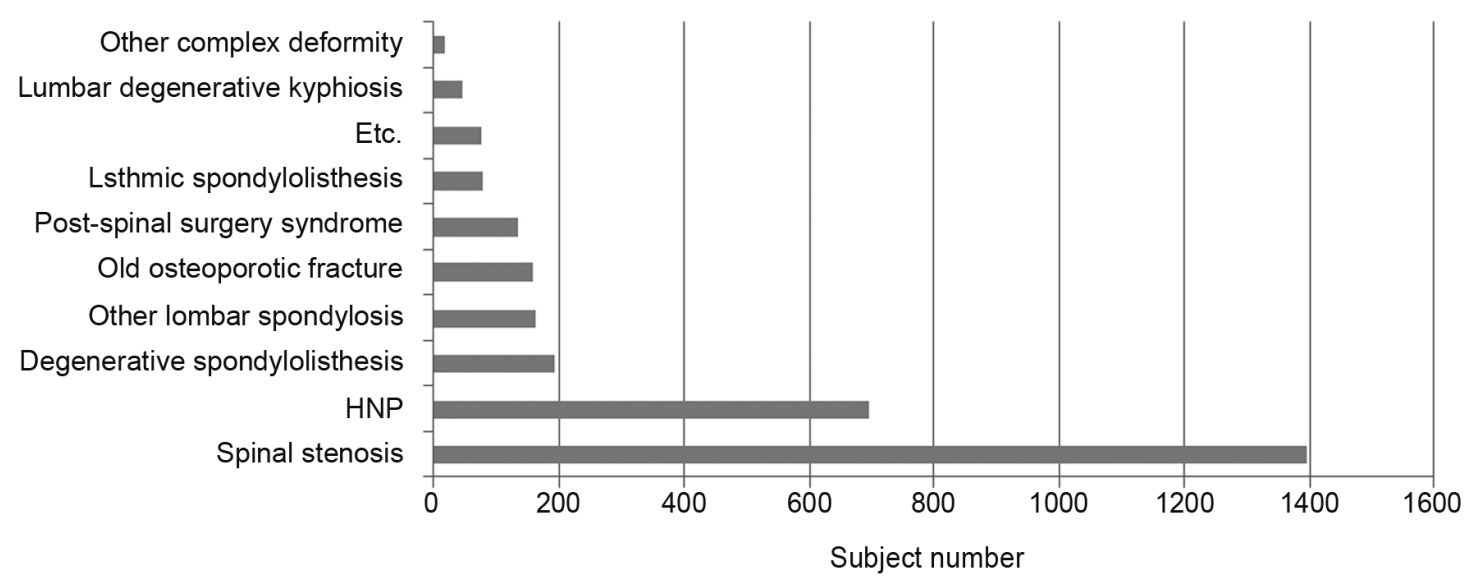

Fig. 1. Causes of chronic low back pain. HNP, herniated nucleus pulposus. 
of patients woke up from sleep at least two times due to LBP (Fig.4). The sleep disturbance was proportional to pain intensity (Fig. 5). And $67.3 \%$ of patients presented moderate to severe pain of the mean pain intensity during the last week (Fig. 6). For LBP, 56.5\% of patients had

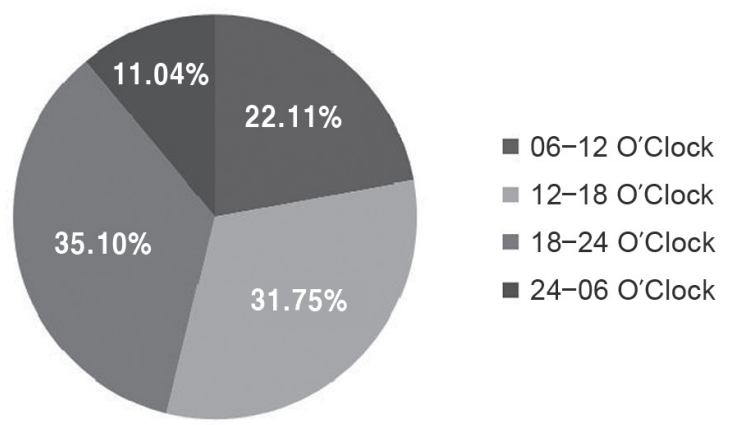

Fig. 3. Pain evoking time-zone during the day.

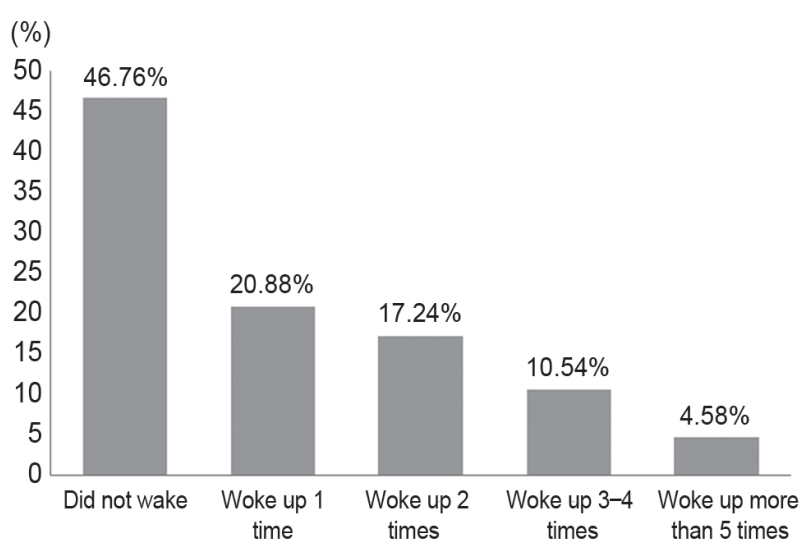

Fig. 4. Sleep disturbance in patients with chronic low back pain.

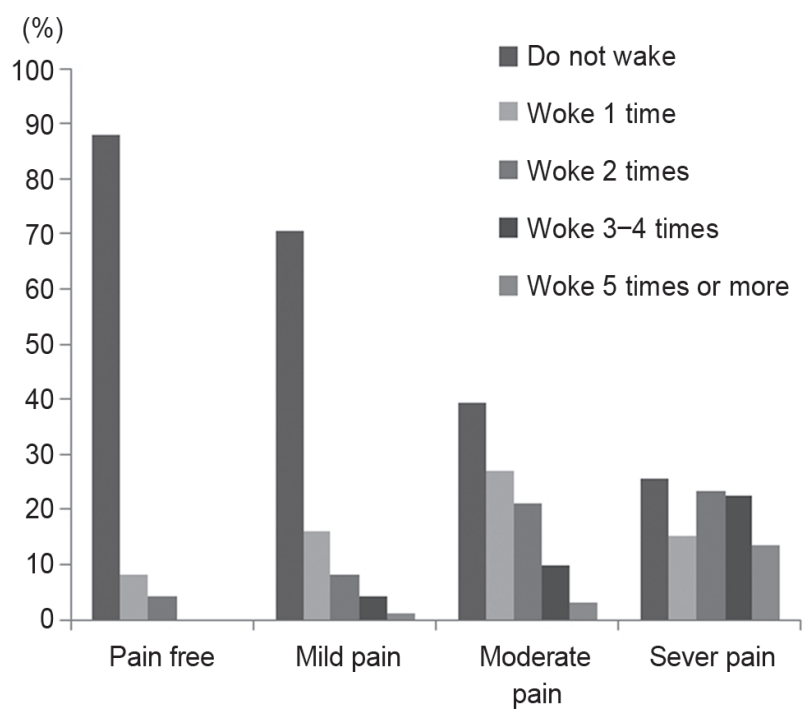

Fig. 5. Mean pain intensity and sleep disturbance. moderate to severe pain; and for radiating pain, $49.8 \%$ of patients had moderate to severe pain. $67.1 \%$ of patients considered that their CLBP was improved after medication; and $32.9 \%$ of patients replied that their pain was not improved (Fig. 7). The ratio of patients who received

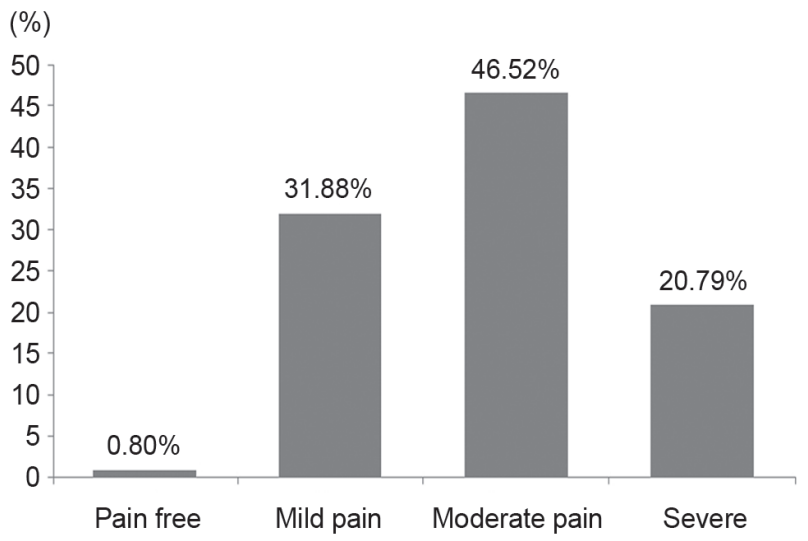

Fig. 6. Mean pain intensity during the last week.

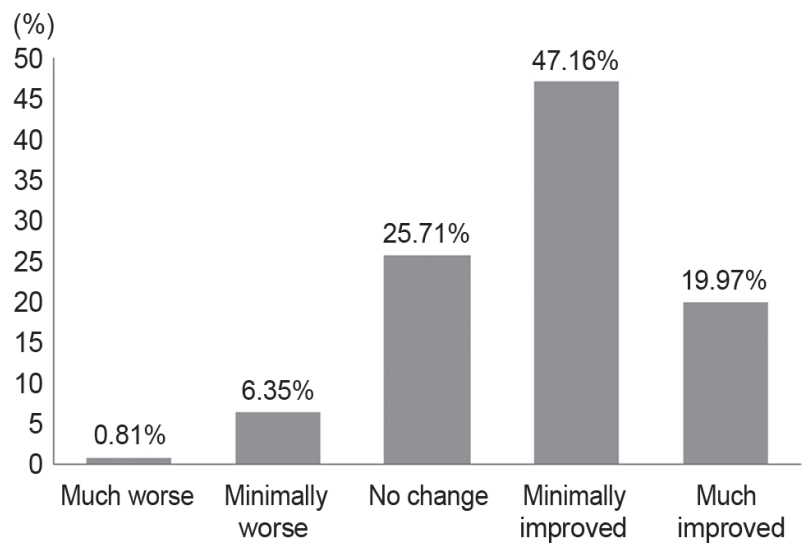

Fig. 7. Pain improvement after medication.

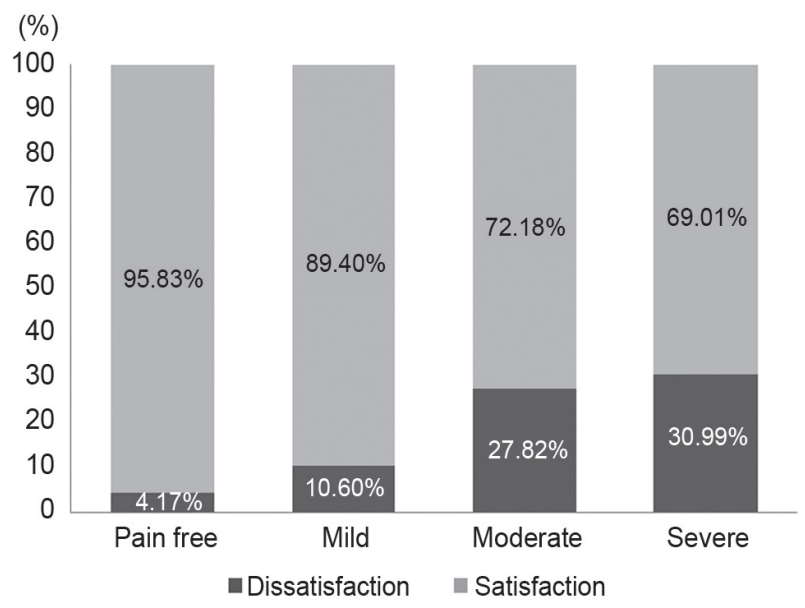

Fig. 8. Satisfaction of current pain management. 
Table 2. The correlation among Pain intensity Korean version Oswestry Disability Index (K-ODI), Satisfaction Short Form 12 Health Survey V2

\begin{tabular}{|c|c|c|c|c|}
\hline Item & K-ODI & Satisfaction & PCS & MCS \\
\hline \multirow[t]{3}{*}{ Pearson correlation score with pain intensity } & 0.61606 & -0.22314 & -0.45673 & -0.37362 \\
\hline & $<0.001$ & $<0.001$ & $<0.001$ & $<0.001$ \\
\hline & 3010 & 2923 & 3010 & 3010 \\
\hline \multirow[t]{3}{*}{ Pearson correlation score with K-ODI } & & -0.2814 & -0.67691 & -0.52496 \\
\hline & & $<0.001$ & $<0.001$ & $<0.001$ \\
\hline & & 2923 & 3010 & 3010 \\
\hline \multirow[t]{3}{*}{ Pearson correlation score with satisfaction } & & & 0.2366 & 0.27047 \\
\hline & & & $<0.001$ & $<0.001$ \\
\hline & & & 2923 & 2923 \\
\hline
\end{tabular}

PCS, physical component score; MCS, mental component score.

Table 3. Comparison of Korean version Oswestry Disability Index score by pain intensity

\begin{tabular}{lccccc} 
Pain intensity $^{\text {a) }}$ & Patients & Mean & Standard deviation & Median & Min-Max \\
Pain free & 25 & 11.04 & 9.22 & 10 & $0-28$ \\
Mild pain & 995 & 24.76 & 14.31 & 22.22 & $0-86$ \\
Moderate pain & $1,450^{b)}$ & 39.16 & 16.22 & 37.78 & $2-88$ \\
Severe pain & 649 & 54.95 & 17.32 & 35.56 & $0-96$ \\
Total & 3,119 & 37.63 & 19.3 & 34 & $0-96$ \\
\hline
\end{tabular}

${ }^{\text {a) }}$ Analysis of variance test; F-value $=505.81, p<0.001$; ${ }^{\text {b) }}$ Missing $=2$.

Table 4. The mean Korean version Oswestry Disability Index (K-ODI) score and correlation with mean pain intensity

\begin{tabular}{lc} 
Item \\
K-ODI \\
$\mathrm{N}$ & 3,119 \\
$\mathrm{Mean} \pm$ standard deviation & $37.63 \pm 19.3$ \\
Median & 34 \\
\hline Minimun-maximum & $0-96$ \\
\hline Mean pain intensity vs. K-ODI & \\
\hline Correlation coefficient & 0.60873 \\
\hline -value & $<0.001$ \\
\hline
\end{tabular}

a)Pearson's correlation coefficient.

treatment other than medication (injection therapy, physical therapy, therapeutic exercise) was $43.4 \%$. Most patients $(77.2 \%)$ were satisfied with their current pain management, but $22.8 \%$ of patients were not satisfied. The dissatisfaction percentage was higher as the pain severity grew (Fig. 8). The correlation between mean pain intensity and satisfaction with current pain management was statistically significant. This indicated that as pain intensity increased, satisfaction level significantly decreased
(Table 2).

The mean K-ODI score of all patients was 37.63. A comparison of K-ODI scores of the four-group levels (divided by mean pain intensity) suggested that difference was statistically significant among each group (ANOVA test, $p 9<0.001$ ) (Table 3). Also the correlation coefficient between the mean pain intensity and the mean K-ODI score showed highly positive correlation (Pearson correlation $=0.61$ ) (Table 4). Hence, the more severe the pain, the higher was the disability in the low back.

The mean PCS was 36.25 , and the mean MCS was 41.77. The results of SF-12v2 were negatively correlated with mean pain intensity and K-ODI score. In addition, there was a positive correlation between SF-12v2 and patient's satisfaction on current pain management. However, SF-12v2 did not show a significant correlation with K-ODI (Table 2).

\section{Doctor's assessment}

Most doctors reported that current medications were NSAIDs $(1,724,55.24 \%)$ and tramadol/acetaminophen extended release (713 cases, $22.85 \%$ ). And $61.6 \%$ of doc- 
Table 5. The conformity between patients and doctors in pain assessment

\begin{tabular}{|c|c|c|c|c|c|}
\hline \multirow{2}{*}{ Patients in pain assessment } & \multicolumn{5}{|c|}{ Doctors in pain assessment } \\
\hline & Pain free & Mild & Moderate & Severe & Total \\
\hline Pain free & 6 & 13 & 5 & 1 & 25 \\
\hline Mild & 4 & 600 & 321 & 60 & 985 \\
\hline Moderate & 1 & 483 & 800 & 133 & 1,417 \\
\hline Severe & 0 & 67 & 352 & 208 & 627 \\
\hline Total & 11 & 1,163 & 1,478 & 402 & 3,054 \\
\hline
\end{tabular}

Correlation analysis Kappa $=0.2463, p<0.001$. Missing=67.

tors replied that they will maintain the current medication in response to a question of 'what analgesic will you prescribe next.' $17.5 \%$ of doctors had administrated a procedure in the past two weeks for CLBP management. In CLBP character assessed by doctors, $61.67 \%$ of patients had LBP with radiating pain and $38.33 \%$ had LBP without radiating pain. In pain intensity assessed by doctors, $61.6 \%$ of patients had moderate to severe LBP. The conformity between patients and doctors in pain assessment revealed that doctors were likely to underestimate a patient's pain intensity $(\mathrm{K}=0.2463)$ (Table 5$)$. $68.1 \%$ of doctors considered that the pain was improved after medication. The assessment of pain improvement between doctors and patients was similar.

\section{Discussion}

In our study, $43.5 \%$ of patients had suffered low back pain for more than two years. In a study that investigated treatment duration for work-related LBP in Korea [10], 70.7\% of subjects were treated for LBP for more than three months, and $20.5 \%$ of subjects were treated for more than two years. In a survey of chronic pain in Europe [11], almost $60 \%$ of subjects had pain for more than two years. There is wide variance among these studies, but CLBP is often prolonged for more than two years. Alsaadi et al. [12] presented that prevalence of sleep disturbance in patients with LBP was 58.7\%, and that the intensity of back pain was weakly associated with sleep disturbance. These results were different from our results $(32.4 \%)$. The difference may be because our results did not include acute LBP.

$67.3 \%$ of patients presented more than moderate pain in our study. Some studies of chronic pain, not CLBP only, reported that $80 \%$ to $100 \%$ of patients with chronic pain presented moderate to severe pain $[11,13]$. It is important that most CLBP patients complain of moderate or severe pain.

The body of evidence for chronic LBP has advanced with the development of reliable and valid patient selfreported outcome measures. However, it is often difficult to make comparisons between published studies, because different outcome measures have been utilized to assess pain, function and quality of life [14]. We sought to answer the clinical questions of how pain characteristics affected the assessment of pain intensity, patient's satisfaction and quality of life. In regard to pain management, most patients (77.2\%) were satisfied with current pain management; and the relationship between pain intensity and patient satisfaction was inversely related. Regarding the impact of CLBP on quality of life, some health status surveys showed that the mean PCS was 47.1, in people whose age was between 55 and 64 years; and the mean PCS was not below 40, in people over 65 years of age. Similarly, the mean MCS was 53.9, for those whose age was between 55 and 64 years; and the mean MCS was not below 50, in people whose age was over 65 years [9]. On the other hand, mean PCS and MCS in our study were 36 and 41.5, respectively. As compared with health status for CLBP generally, our results revealed lower health status than these PCS and MCS. The disability and quality of life decreased when pain was increased; and relationship between disability and quality of life was inverse. These results were similar to other studies $[11,15]$.

The doctors' assessment of pain intensity was lower than those of the patients' assessment. But there was no significant difference between doctors and patients evaluations of the mean pain intensity. Also there was no difference in assessment of pain improvement after medica- 
tion between doctors and patients.

This study was limited to patients in outpatient clinics; and the results are not necessarily generalized to other settings. In addition, results may not be representative at a national level because of the limited number of participating centers.

\section{Conclusions}

The present survey has suggested that CLBP increases negative aspect of quality of life. Of total $22.8 \%$ of the patients were not satisfied with current pain management. Such needs to be taken more seriously by doctors for improvement in satisfaction and quality of life in patients with CLBP.

\section{Conflict of Interest}

No potential conflict of interest relevant to this article was reported.

\section{Supplementary Materials}

Suppl. 1. Korean version of Oswestry Disability Index (KODI). Supplementary material can be found via http:// www.asianspinejournal.org/src/sm/asj-8-346-s001.pdf Suppl. 2. Korean version of Short Form 12 Health Survey V2 (SF-12v2). Supplementary material can be found via http://www.asianspinejournal.org/src/sm/asj-8-346-s002. pdf

\section{References}

1. Ruoff GE, Rosenthal N, Jordan D, Karim R, Kamin M; Protocol CAPSS-112 Study Group. Tramadol/acetaminophen combination tablets for the treatment of chronic lower back pain: a multicenter, randomized, double-blind, placebo-controlled outpatient study. Clin Ther 2003;25:1123-41.

2. Deyo RA, Weinstein JN. Low back pain. N Engl J Med 2001;344:363-70.

3. Von Korff M, Saunders K. The course of back pain in primary care. Spine (Phila Pa 1976) 1996;21:2833-7.

4. Andersson GB. Epidemiological features of chronic low-back pain. Lancet 1999;354:581-5.
5. Ashburn MA, Staats PS. Management of chronic pain. Lancet 1999;353:1865-9.

6. Carr DB, Jacox AK, Chapman CR. Acute pain management: operative or medical procedures and trauma. In: Clinical Practice Guideline. Rockville, MD: Agency for Health Care Policy and Research, Public Health Service, U.S. Department of Health and Human Services; 1992. AHCPR Pub. No. 92-0032.

7. Fairbank JC, Couper J, Davies JB, O’Brien JP. The Oswestry low back pain disability questionnaire. Physiotherapy 1980;66:271-3.

8. Fisher K, Johnston M. Validation of the Oswestry low back pain disability questionnaire, its sensitivity as a measure of change following treatment and its relationship with other aspects of the chronic pain experience. Physiother Theory Pract 1997;13:67-80.

9. Office of Public Health Assessment Center for Health Data Utah Department of Health. 2001 Utah Health Status Survey report Health Status in Utah: The Medical Outcomes Study SF-12 [Internet]. Salt Lake City, UT: Office of Public Health Assessment Center for Health Data Utah Department of Health; 2004 [cited 2014 Apr 20]. Available from: http://health.utah.gov/ opha/publications/2001hss/sf12/2001HSS_sf12.htm.

10. Kim HS, Choi JW, Chang SH, Lee KS, Oh JY. Treatment duration and cost of work-related low back pain in Korea. J Korean Med Sci 2005;20:127-31.

11. Breivik H, Collett B, Ventafridda V, Cohen R, Gallacher D. Survey of chronic pain in Europe: prevalence, impact on daily life, and treatment. Eur J Pain 2006;10:287-333.

12. Alsaadi SM, McAuley JH, Hush JM, Maher CG. Prevalence of sleep disturbance in patients with low back pain. Eur Spine J 2011;20:737-43.

13. Moulin DE, Clark AJ, Speechley M, Morley-Forster PK. Chronic pain in Canada: prevalence, treatment, impact and the role of opioid analgesia. Pain Res Manag 2002;7:179-84.

14. Chapman JR, Norvell DC, Hermsmeyer JT, et al. Evaluating common outcomes for measuring treatment success for chronic low back pain. Spine (Phila Pa 1976) 2011;36(21 Suppl):S54-68.

15. Harker J, Reid KJ, Bekkering GE, et al. Epidemiology of chronic pain in denmark and sweden. Pain Res Treat 2012;2012:371248. 